\title{
RESEARCH
}

\section{Mapping in the Region of Danforth's Short Tail and the Localization of Tail Length Modifiers}

\author{
Jane B. Alfred, ${ }^{1,5}$ Kellie Rance, ${ }^{2}$ Benjamin A. Taylor, ${ }^{3}$ Sandra J. Phillips, ${ }^{3}$ \\ Catherine M. Abbott, ${ }^{4}$ and lan J. Jackson ${ }^{1}$
}

\begin{abstract}
${ }^{1}$ Medical Research Council Human Genetics Unit, Western General Hospital, Edinburgh, EH4 2XU, UK; ${ }^{2}$ Institute of Cell, Animal, and Population Biology, University of Edinburgh, King's Buildings, Edinburgh, EH9 3JT, UK; ${ }^{3}$ The Jackson Laboratory, Bar Harbor, Maine 04609; ${ }^{4}$ Human Genetics Unit, Department of Medicine, University of Edinburgh, Molecular Medicine Centre, Western General Hospital, Edinburgh,
\end{abstract} EH4 2XU, UK

\begin{abstract}
We have used an interspecific backcross to generate a detailed genetic map around the mouse tail and kidney developmental mutation Danforth's short tail (Sd). The map includes 14 simple sequence repeat (SSR) markers and four genes in a 5-cM region encompassing Sd. In addition we have used a DNA pooling approach to carry out a genome scan to localize quantitative trait loci (QTL) that modify the tail length of Sd progeny of the backcross. This has allowed us to identify a major QTL on chromosome 10 in the region of nodal and three other putative tail length QTL on chromosomes 1, 9, and 18.
\end{abstract}

Danforth's short tail $(S d)$ is a semidominant mouse mutation that affects the development of the vertebral column and the urogenital organs (Dunn et al. 1940; Gluecksohn-Schoenheimer 1943). Homozygous ( $S d S d$ ) mice survive to term but die shortly after birth usually because of bilateral renal agenesis. Sacral and lumbar vertebrae fail to develop normally in these mice, giving rise to a characteristic tailless phenotype. In addition, homozygous mice lack external urogenital and anal openings. Mice heterozygous for $S d(S d+)$ have a variable phenotype and are distinguished by their shortened and often misshapen tails. Renal abnormalities in $S d+$ mice range from unilateral renal agenesis to mildly affected kidneys, with the severity of the phenotype in some studies determining the viability of heterozygotes (GluecksohnWaelsch and Rota 1963). The notochord degenerates in heterozygous and homozygous $S d$ embryos from day 10 of development, causing the establishment of the floor plate to fail and the aberrant expression of genes that determine dorsal/ventral polarity in the developing neural tube (Gruneberg 1953; Placzek et al. 1991; Dietrich et al. 1993). The basis of the urogenital defects in $S d$ mice remains unknown.

The $S d$ mutation maps to proximal mouse chro-

${ }^{5}$ Corresponding author.

E-MAIt Janea@hgu.mrc.ac.uk; FAX 44-131-343-2620. mosome 2 (Beechey and Searle 1980), where several other cloned genes of interest have been mapped (Siracusa et al. 1996). To assess the candidacy of a number of these genes and to provide a basis for positional cloning, we generated an intersubspecific backcross segregating for $S d$.

In generating the backcross we noted that tail lengths in the $\mathrm{N}_{2} S d$ offspring were shorter and of greater variability than those of the $\mathrm{F}_{1}$ generation. As the $F_{1}$ animals are largely genetically identical, whereas the backcross progeny have segregated loci of the two parental strains, it is possible that the variation in tail length is attributable to the segregation of loci throughout the genome that modify the $S d$ phenotype. Phenotypic traits, such as tail length, which are determined by the segregation of multiple loci, are referred to as complex or quantitative traits. The loci that determine the variation in these traits are known as quantitative trait loci (QTL) (Lander and Botstein 1989).

To identify QTL affecting variation in the tail length of $S d$ mice we carried out a genome-wide scan using polymorphic simple sequence repeat markers amplified from phenotypically pooled sources of DNA (Darvasi and Soller 1994; Taylor et al. 1994). This approach has identified three loci, which together contribute $-30 \%$ of the variance in the tail length of $S d$ mice. 


\section{MAPPING AND MODIFIERS OF DANFORTH'S SHORT TAIL}

\section{RESULTS}

\section{Linkage Mapping in the Region of Sd}

For linkage mapping, we generated a 336-progeny intersubspecific backcross using mice derived from the CAST/Ei strain of Mus musculus castaneus. On this genetic background $F_{1}$ mice appeared healthy and of normal viability. The ratio of wild-type $(49.8 \%)$ to heterozygous $(50.2 \%)$ mice showed $S d$ to be fully penetrant in this backcross. One of the advantages of using the $M . m$. castaneus subspecies in generating backcrosses is that progeny can be derived from both male and female meioses. In our backcross, $233 \mathrm{~N}_{2}$ mice were derived from male meioses and 103 from female.

To map $S d$ the first 100 progeny of the backcross were typed for the markers D2Mit1, D2Mit31, Pax8, D2Mit79, and D2Mit7. These markers were chosen on the basis of their position in the consensus map (Siracusa et al. 1996) as markers that potentially flanked $S d$ and were close enough to exclude double recombinants. $\operatorname{Pax} 8$ was included in the analysis as a candidate for the mutation. Data generated from these mice allowed us to establish D2Mit31 and D2Mit7 as markers that flanked $S d$. Accordingly, we typed 236 subsequent backcross mice for these two markers and those recombinant in this interval for Pax8. Once Pax 8 had been excluded as a candidate gene, the final $56 \mathrm{~N}_{2}$ mice were typed for only D2Mit31 and D2Mit7. The 18 mice recombinant between D2Mit31 and Pax8 or D2Mit31 and D2Mit7, we mapped in more detail using the following markers: D2Mit119, D2Mit149 (Vim), D2Mit118, D2Mit267, D2Ucl2, D2Mit464, D2Mit362, Bmi1, D2Mit80, D2Mit6, D2Mit363, D2Mit364, D2Mit416, Il $1 r n$, and D2Mit292. Haplotype data are shown in Figure 1.

The deduced order of markers and genes based on minimizing the number of double recombinants in the region of $S d$ is illustrated in Figure 2 . The numbers of recombinants between each marker pair are as follows: D2Mit1-7/ $100-D 2 M i t 31-0 / 336-$ D2Mit119-2/336-D2Mit118-0/ 336-D2Mit149 (Vim)-1/336D2Ucl2-4/336-D2Mit267-2/
336-D2Mit362-0/336-D2Mit464-0/336-Bmi1-1/ 336-Sd-2/336-D2Mit80-0/336-D2Mit6-0/336D2Mit416-0/336-D2Mit363-0/336-D2Mit364-0/ 336-Il1rn-1/280-D2Mit292-0/280-D2Mit79-0/280Pax8-31/280-D2Mit7. The order of markers as we have placed them on the map is in broad agreement with their order on the chromosome 2 consensus map (Siracusa et al. 1996).

Figure 2 shows a number of genes in the region that have been mapped onto the backcross and excluded as candidates for the mutation. For some time, the paired box gene family member $\operatorname{Pax} 8$ was considered to be a strong candidate for $S d$ on the basis of its map position and its spatiotemporal expression patterns during development (Plachov et al. 1990). Previously, the candidacy of Pax 8 had been excluded by mapping data compiled from a number of intraspecific crosses (Koseki et al. 1993). However, Pax 8 was not mapped in a cross segregating for $S d$. By mapping Pax8 onto a backcross in which $S d$ is segregating, we show conclusively that a mutation in Pax 8 is not responsible for $S d$. Three other genes in the region, Vim, Il1m, and Bmi1, have also been excluded because our data show that one or more recombination events have occurred between them and $S d$.

By typing the backcross for a number of simple sequence repeat (SSR) markers, we have established markers that closely flank Sd:D2Mit362 and

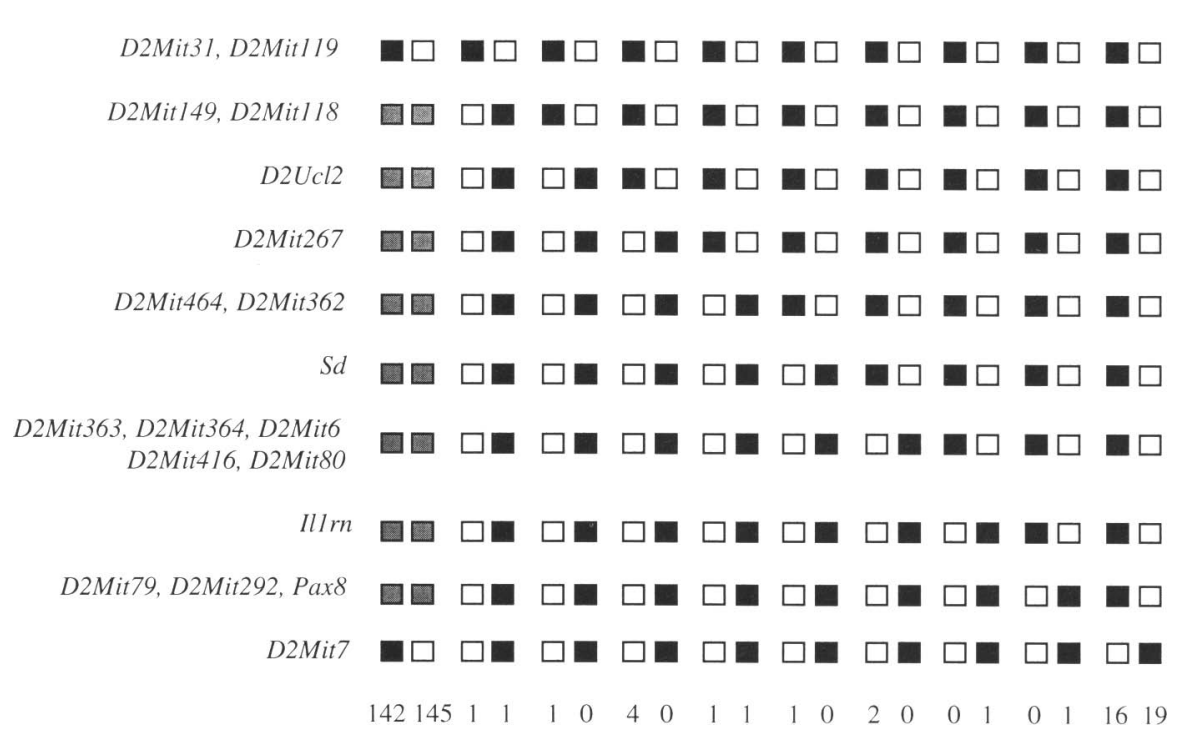

Figure 1 Haplotypes from mice recombinant between D2Mit31 and D2Mit7. Numbers at the base of each column indicate the number of mice that were recombinant in the $F_{1}$ parent between the markers shown at left. (Black boxes) The CAST alleles; (open boxes) the CBA alleles, on the $F_{1}$-derived chromosome; (shaded boxes) loci that were not typed in animals nonrecombinant in the D2Mit31 to D2Mit7 interval. 
ALFRED ET AL.

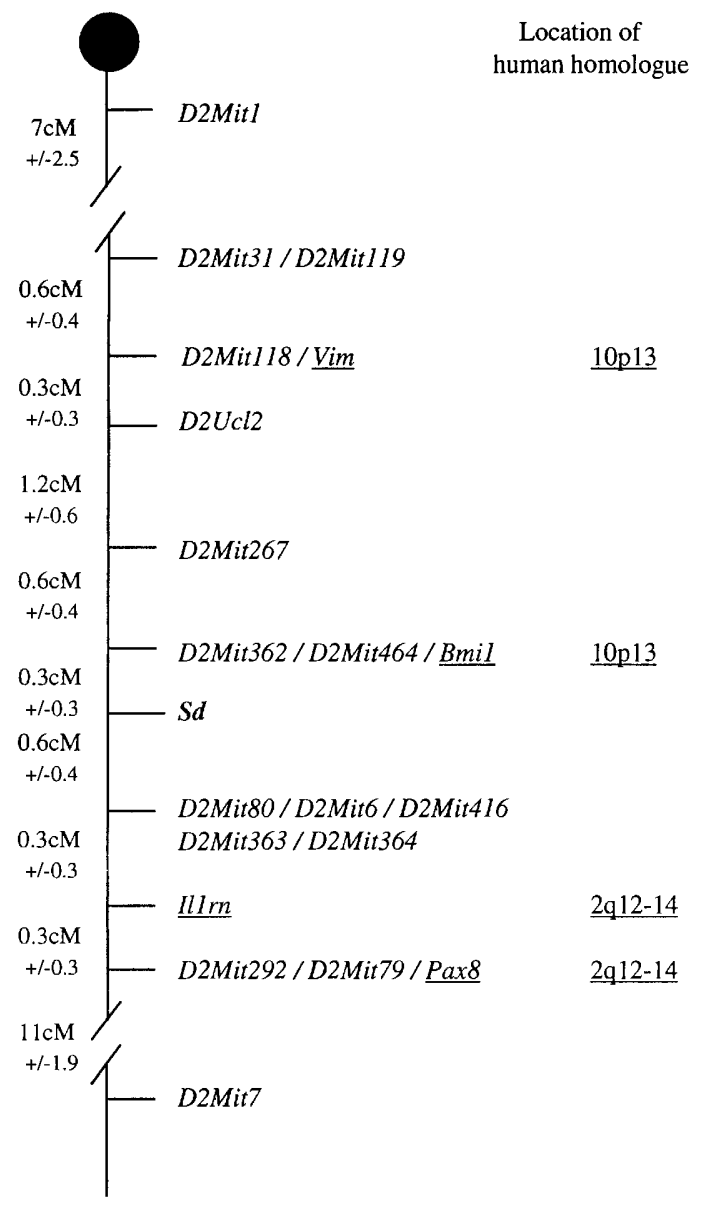

Figure 2 Genetic map in the region of Sd. Mapped loci are listed to the right of the chromosome and distances between loci in centiMorgan (cM) to the left. Genes with mapped human homologs are underlined, their location in the human genome taken from Siracusa et al. (1996).

D2Mit464, which map $0.3 \mathrm{cM}$ proximal to $S d$, and D2Mit80, D2Mit6, D2Mit416, D2Mit363, and D2Mit364, which map $0.6 \mathrm{cM}$ distal to $S d$. These closest markers to $S d$ provide an entry point for positional cloning. We also mapped the panel of markers used for fine mapping to a number of backcross progeny nonrecombinant between D2Mit31 and D2Mit7, and therefore confirmed their location to chromosome 2 . The marker D2Mit464 uniquely amplified from the $S d$ allele a product that was different in size from that amplified from the CBA allele, indicating the presence of a congenic interval flanking $S d$, which is not derived from the CBA genome.

\section{Detection and Mapping of Modifiers of Sd}

We measured the tail lengths of $\mathrm{N}_{2}$ mice at 24 days of age and found that the tail length distributions of wild-type and mutant mice did not overlap (Fig. 3). In an analysis of variance (see Methods), we found the difference in the distribution of tail lengths between the two populations to be highly significant $(P<0.0001)$, showing that the $S d$ animals had a significantly wider range of tail length compared to their wild-type littermates. Despite the bimodal appearance of tail length distribution in the $S d$ population, there is no statistical evidence to support such a distribution.

We were interested in identifying the genetic factors that caused the increased tail length variance. To minimize time and effort in the mapping of these trait-modifying loci we used a phenotypic pooling approach (Taylor et al. 1994; Taylor and Phillips 1996). This strategy entails pooling DNA from a segregating population according to phenotype, before marker analysis, rendering it unnecessary to genetically type each animal in a genomewide scan. Although this method was developed originally to facilitate the mapping of single locus mutations, we have modified it to map traitmodifying loci in a backcross population.

We pooled DNA from $S d$ mice whose tail lengths were in the longest and shortest $20 \%$ of the backcross population and used a panel of 65 markers, chosen to scan $94 \%$ of the autosomal genome, to examine the pools (Fig. 4). A marker that is unlinked to any locus that affects tail length in $S d$ mice should amplify the two alleles from the two DNA pools with the same relative ratio of 3:1, CBA:CAST. A marker that is linked to a locus that modifies $S d$ tail length will show a difference in the ratio of amplification products between the two pools, tending

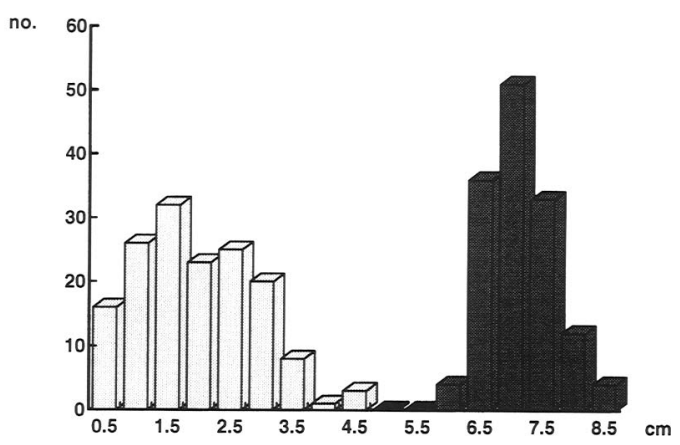

Figure 3 Distribution of tail lengths in backcross progeny at 24 days of age. Light-shaded bars represent tail lengths of $S d$ progeny $(n=147)$; dark-shaded bars represent tail lengths of wild-type progeny $(n=144)$. The difference in variance between the two populations is highly significant $(P<0.0001)$. 
MAPPING AND MODIFIERS OF DANFORTH'S SHORT TAIL

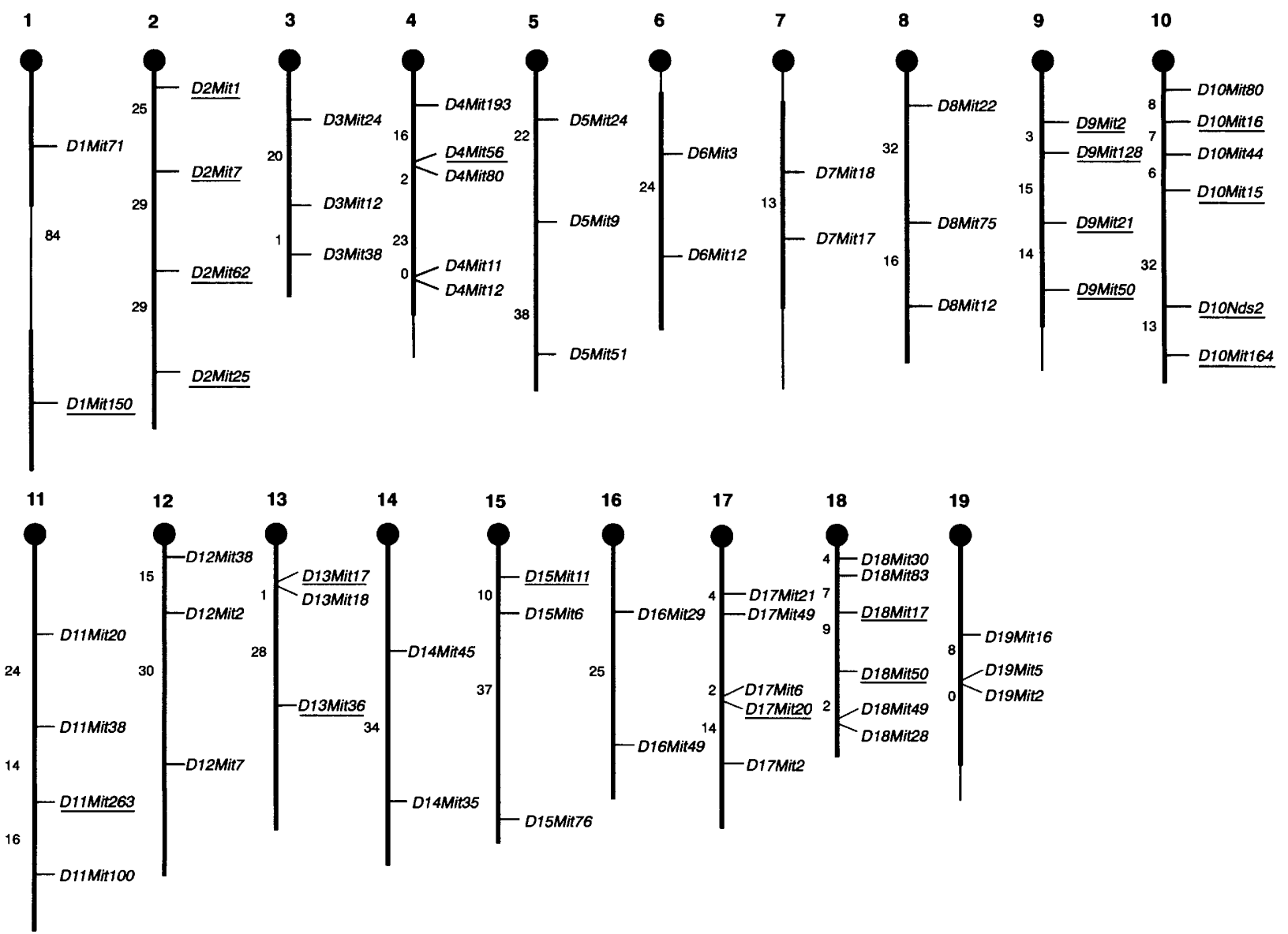

Figure 4 The chromosomal location of the markers selected for the analysis of phenotypic pools. Marker positions were obtained from the microsatellite map distributed by the MIT Genome Center. Vertical lines indicate regions within $20 \mathrm{cM}$ of a marker. Numbers to the left of each chromosome indicate the genetic distance between markers in centiMorgans. Underlined markers are those that showed a distortion in the ratio of CAST/CBA alleles in one or both phenotypic pools.

toward entirely CBA in one pool and toward a 1:1 ratio in the other.

From this survey, we identified four regions on chromosomes $1,9,10$, and 18 that showed a distortion in the ratio of alleles at more than one locus (markers on chromosome 2 showed a reduction in CAST alleles in both pools, as expected for markers linked to $S d$ ). We typed DNA from each animal contributing to the pools with a selection of additional SSR markers from the four regions to locate more definitely the QTL detected by the scan (Table 1). As the central region of chromosome 1 was not scanned by the marker panel, in addition we typed individuals with markers from this region. From inspection of the haplotypes, we observed an association of the CAST allele with the long-tail pool and a reciprocal association of the $\mathrm{CBA}$ alleles with the short-tail pool, as expected from our observation of the $F_{1}$ phenotype, where tail length increases with the presence of CAST chromosomes. The reciprocal peaks of contribution of CAST or CBA alleles to the two pools provided an indication of the approximate likeliest locations of trait-modifying loci, as shown in Table 1 . We then typed all $S d$ animals whose phenotype had been assayed at 24 days $(n=152)$ from the backcross using the peak markers and those flanking the peaks at $\sim 10 \mathrm{cM}$. In addition, for the markers on chromosome 10 we typed wildtype progeny at the same age $(n=148)$ to assess whether modifying loci also affected wild-type tail length.

The gene encoding the transforming growth factor- $\beta$ (TGF $\beta$ ) superfamily member nodal maps in the region of one of the putative tail lengthmodifying loci on chromosome 10 and is required for axial formation (Conlon et al. 1994). Embryos 


\begin{tabular}{|c|c|c|c|}
\hline \multirow[b]{2}{*}{ Chromosome } & \multirow[b]{2}{*}{ Markers } & \multicolumn{2}{|c|}{ Percent } \\
\hline & & $\begin{array}{l}\text { CAST } \\
\text { long-tail pool }\end{array}$ & $\begin{array}{l}\text { CBA } \\
\text { short-tail poo }\end{array}$ \\
\hline \multirow[t]{3}{*}{1} & D1Mit71 & 60 & 53 \\
\hline & D1Mit257 & 60 & 60 \\
\hline & D1Mit37 & 63 & 60 \\
\hline \multirow[t]{5}{*}{9} & D9Mit160 & 77 & 60 \\
\hline & D9Mit2 & 83 & 37 \\
\hline & D9Mit23 & 77 & 64 \\
\hline & D9Mit21 & 77 & 63 \\
\hline & D9Mit260 & 80 & 60 \\
\hline \multirow[t]{6}{*}{10} & D10Mit80 & 67 & 70 \\
\hline & D10Mit105 & 87 & 70 \\
\hline & nodal & 80 & 73 \\
\hline & D10Mit31 & 70 & 73 \\
\hline & D10Mit21 & 67 & 70 \\
\hline & D10Mit121 & 60 & 63 \\
\hline \multirow[t]{4}{*}{18} & D18Mit97 & 53 & 60 \\
\hline & D18Mit140 & 60 & 60 \\
\hline & D18Mit2 & 57 & 76 \\
\hline & D18Mit42 & 63 & 80 \\
\hline
\end{tabular}

homozygous for a loss-of-function mutation at nodal die early in development due to an inability to differentiate mesoderm from ectoderm during gastrulation (Zhou et al. 1993). We considered nodal to be a candidate for the putative tail length modifier on chromosome 10 and therefore developed a PCR assay for genotype at the locus by amplifying across a small intron. Using this assay we typed all animals from the backcross for nodal.

Analysis of the haplotype data was performed using the statistical model (see Methods), which was - fitted at 1-cM intervals for chromosomes 1, 9, and 18 and at $0.5-\mathrm{cM}$ intervals for chromosome 10 . Lod score profiles generated from data for chromosomes $1,9,10$, and 18 are shown in Figure 5. Using a multiple regression analysis, a maximum lod score of 7.8 was obtained in the interval between the markers D10Mit105 and nodal, $5.8 \mathrm{cM}$ distal from D10Mit105, and $4.1 \mathrm{cM}$ proximal to nodal. This modifier locus accounts for $18.9 \%$ of tail length variance in $S d$ mice. A 95\% confidence interval, defined by a one value drop of the maximum lod score, encompasses a 13-cM interval of chromosome 10 within which is included nodal, the putative candidate for the modifier (Fig. 5a). Thus, our data indicate the presence of a QTL on chromosome 10 that, in the presence of $S d$, modifies tail length. The separate analysis of genotypes of wild-type mice produced a maximum lod score of 0.8 for the chromosome 10 QTL. Therefore, we are unable to detect a tail length QTL on chromosome 10 in wild-type animals.

A second putative tail length QTL was located on chromosome 18, $1 \mathrm{cM}$ from the most distal marker, D18Mit42. The maximum lod score of 2.7 is suggestive of linkage to a putative QTL (Kruglyak and Lander 1995). This locus accounts for $7.1 \%$ of phenotypic variance, and the $95 \%$ confidence interval encompasses a 17-cM region of chromosome 18 (Fig. 5b).

We also found suggestive linkage to a putative QTL on chromosome 1 (Fig. 5c), with a peak in the lod score of 1.9 at $9 \mathrm{cM}$ distal to D1Mit257 and $19 \mathrm{cM}$ proximal to D1Mit73. The $95 \%$ confidence interval for this QTL spans a distance of 38.5 $\mathrm{cM}$ and the locus accounts for $4.1 \%$ of phenotypic variance.

Further analysis of haplotype data from chromosome 9 indicated a maximum lod score of 1.8 , marginally below that considered to be suggestive 
MAPPING AND MODIFIERS OF DANFORTH'S SHORT TAIL

chromosome 10
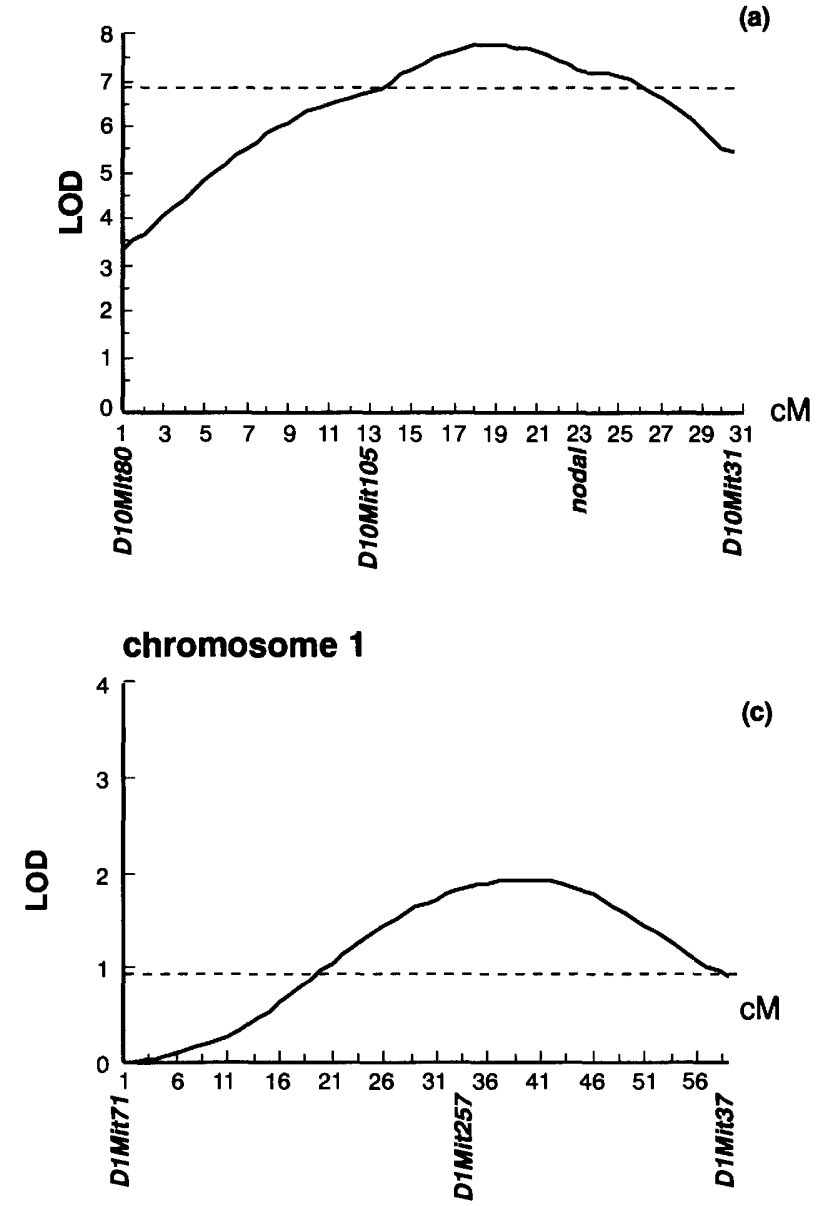

chromosome 18

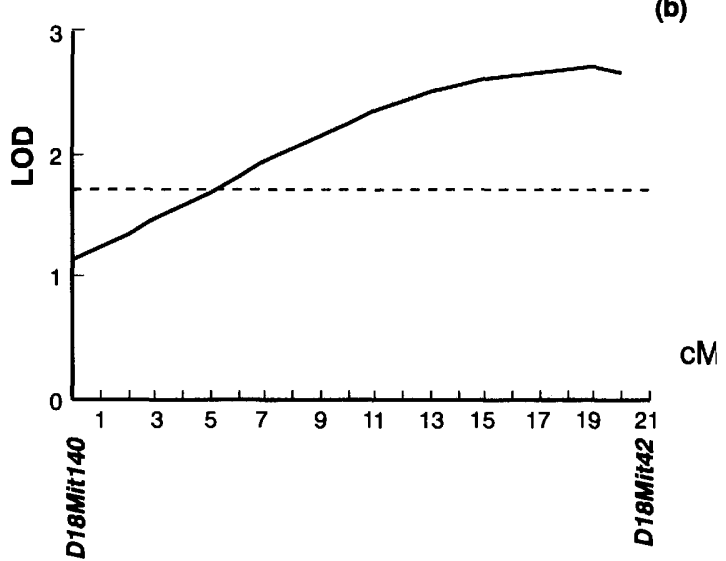

chromosome 9

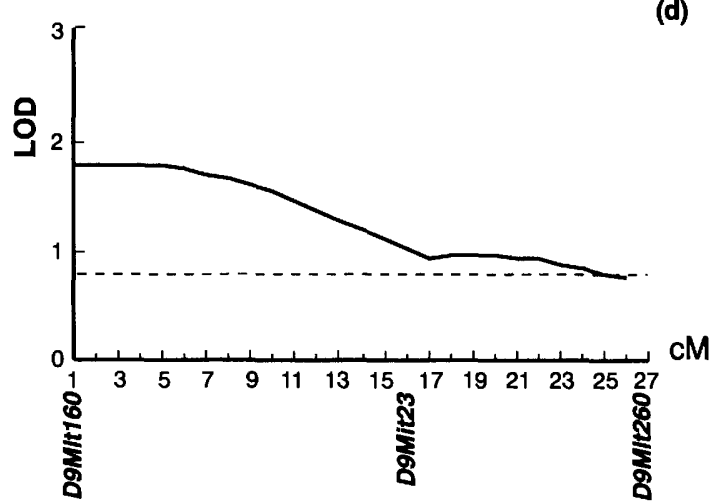

Figure 5 Lod score profiles for tail length modifier loci. The genetic intervals used to fit the data are indicated on the horizontal axis, and the markers used in the analysis are below this axis. The lod scores obtained are on the vertical axis. A one-lod drop from the peak of the profile is indicated by the horizontal broken line.

of linkage. Nevertheless, further analysis of this chromosome in a larger backcross might be fruitful.

\section{DISCUSSION}

\section{Linkage Mapping}

We have generated the first detailed map on proximal mouse chromosome 2 in the region of $S d$ using a single intersubspecific backcross segregating for the mutation. Our results exclude $\operatorname{Pax} 8$ as a candidate gene for the mutation, in agreement with data published previously by Koseki et al. (1993). We also genetically exclude three other candidate cDNAs: Vim, encoding the intermediate filament protein vimentin; $I l 1 m$, the interleukin-1 receptor antagonist; and Bmi1, which encodes a homolog of the Drosophila polycomb gene. Bmi1 is only excluded by a single recombinant chromosome, and it is possible that this was an intragenic recombination event separating the $S d$ mutation from the 3 '-untranslated region used as a probe. Both null mutations and overexpressing transgenic mice have been reported for Bmi1, and neither have an $S d$-like phenotype (van der Lugt et al. 1994; Alkema et al. 1995), but it is still conceivable that a gain-of-function mutation within Bmi1 could be responsible for the $S d$ phenotype. However, in the absence of alternative candidate genes we have established two sets of flanking markers that provide a framework from which to initiate the positional cloning of $S d$.

Searches for additional candidates could come from work on the homologous region of the human genome. However, at the moment it is not possible to identify this region, as $S d$ is flanked on one side by markers that map to human chromosome $10 \mathrm{p} 13$ and on the other by markers on human 2q12-14 (Siracusa et al. 1996). Nevertheless, we predict that 


\section{ALFRED ET AL.}

the human homolog of $S d$ will map to one of these two human chromosomal segments.

\section{QTL Mapping}

We have carried out a genome-wide scan on pooled sources of DNA to identify putative tail lengthmodifying loci in $S d$ mice, using a method developed originally to map single-locus mutations (Taylor et al. 1994). Data from this scan indicate the presence of QTL on chromosomes 1, 9, 10, and 18. Backcross progeny were typed for a selection of markers across these chromosomes and regression analysis was used to analyze the data. Regression analysis uses a marker interval to estimate QTL effect and position and has been shown to produce results almost indistinguishable from those obtained by the maximum likelihood approach (Lander and Bostein 1989; Haley and Knott 1992). It has allowed us to fit a more complex model to the data by taking into account the phenotypic effects of litter and sex.

Lod scores indicative of linkage to tail-length modifiers were obtained for three of the four chromosomal loci. Our data show the highly significant linkage (lod score of 7.8, $P<0.001$ ) of a tail-length QTL to chromosome 10, in the vicinity of the markers D10Mit105 and nodal. We were unable to detect linkage of this QTL to tail length variation in wildtype progeny. This does not mean that the QTL does not affect wild-type tail length but that any effect may be too small to be detected in this backcross. The effect may be seen only when the developmental pathway leading to tail formation has been "sensitized" by the $S d$ mutation. Our model accounts for a single modifier locus but does not exclude the possibility that the chromosome 10 QTL is composed of two or more closely linked loci.

For chromosome 18 we obtained a lod score of 2.7 for a tail-length QTL close to the marker D18Mit42. This value is suggestive of linkage to a tail-length modifier (Kruglyak and Lander 1995) and places the QTL in the telomeric region of chromosome 18 where few genes have been mapped (Johnson and Davisson 1996).

Linkage of a putative tail length modifier in $S d$ mice to leaden ( $l n$ ) on chromosome 1 has been proposed previously, although no mapping data in support of a QTL have been presented (Wallace 1972, 1976). Our data generated a lod score of 1.9 in the region of D1Mit257, which maps proximal to $\ln$ and places $\ln$ within the 9-cM region that lies between D1Mit257 and the likeliest location for the QTL. Although there may be a QTL that maps to this region, it only contributes $\sim 4 \%$ of the variance and is not the major tail-length modifier of $S d$ mice in our backcross.

Of the four loci analyzed, the chromosome 10 QTL is responsible for the greatest variance in $S d$ tail length. The TGF- $\beta$ superfamily member nodal maps within $4.1 \mathrm{cM}$ of the likeliest location of the chromosome 10 QTL and encodes a signaling molecule essential for axial development and mesoderm differentiation (Zhou et al. 1993; Conlon et al. 1994). Differences in the sequence of the nodal protein between CAST and CBA could underlie subtle changes in the function of the protein, affecting the mechanisms by which it interacts with its receptor, or the stability of the protein itself. In addition or alternatively, there could be slight differences between the two alleles in the temporal or spatial expression patterns of the gene. If nodal was acting in a signaling pathway sensitized by $S d$, whereby the mutation affected the interaction of nodal with other components of the pathway, its modifier effect would be detectable in mutant mice alone. Furthermore, assessment of nodal as a modifier of $S d$ will require genetic crosses to refine the interval containing the chromosome $10 \mathrm{QTL}$, along with comparison of the sequence of the CBA and CAST nodal alleles, and an analysis of their relative timing and levels of expression.

\section{METHODS}

\section{Mice}

Danforth's short tail mice were obtained from MRC Mammalian Genetics Unit, Didcot, UK, where they were maintained as a linkage stock on an outbred background. $S d$ mice were crossed with a $M$. musculus inbred laboratory strain derived from $\mathrm{CBA} / \mathrm{Ca}$ and were maintained on this background for two generations before use in the backcross. $M$. $m$. castaneus mice of the strain CAST/Ei were obtained from Harlan-OLAC Ltd., Bicester, UK. An intersubspecific backcross was generated by mating $S d$ mice to CAST/Ei. $F_{1}$ mice heterozygous for $S d$ were crossed back to the CBA strain to produce backcross progeny.

\section{Phenotypic Analysis}

Backcross progeny were killed 3 days after weaning, at 24 days, and all were dissected to remove their spleens and kidneys. Tail lengths were recorded, along with kidney size and any visible abnormalities. Spleens and tail tips were snap frozen in liquid nitrogen and stored at $-70^{\circ} \mathrm{C}$ before the preparation of DNA.

\section{Genotypic Analysis}

DNA was prepared from spleens that were homogenized in 2 


\section{MAPPING AND MODIFIERS OF DANFORTH'S SHORT TAIL}

$\mathrm{ml}$ of TNES [50 mm Tris- $\mathrm{HCl}(\mathrm{pH} 7.5), 400 \mathrm{~mm} \mathrm{NaCl}, 100 \mathrm{~mm}$ EDTA, $0.5 \%$ SDS] and incubated overnight at $55^{\circ} \mathrm{C}$ with 100 $\mu \mathrm{g} / \mathrm{ml}$ of proteinase $\mathrm{K}$. An equal volume of $2.6 \mathrm{M} \mathrm{NaCl}$ was added to each sample before centrifugation at $12,000 \mathrm{~g}$ for 5 min. DNA was precipitated from the supernatant with ethanol, washed once in $70 \%$ ethanol, and resuspended in $500 \mu 1$ TE [10 mM Tris- $\mathrm{HCl}$ (pH 8.0), 1 mM EDTA].

The mapping of MIT markers was performed using oligonucleotides from Research Genetics (Huntsville, AL), which amplify SSR polymorphisms, using the conditions described in Dietrich et al. (1992). D2UCl2 was amplified as detailed in Malas et al. (1996). In all cases, amplification by PCR was carried out in a $25-\mu$ l volume using $100 \mathrm{ng}$ of DNA, $50 \mathrm{ng}$ of each primer, $1.5 \mathrm{mM} \mathrm{MgCl}, 2.5 \mathrm{mM}$ of each dNTP, and 0.5 units of AmpliTaq or AmpliTaq Gold polymerase (Perkin Elmer). Reactions were performed on an Omnigene (Hybaid) thermal cycler. PCR products of $300 \mathrm{bp}$ or smaller were resolved in $4 \%$ NuSieve agarose (FMC Bioproducts). For the mapping of Pax8 (Plachov et al. 1990), a 438-bp fragment from the $3^{\prime}$-untranslated region was amplified with the primers 5'-GTCATGGGGACATGTGGAAG-3' and 5'-CCTAGGCTGTGATTGTAGGGC- $3^{\prime}$ under the following conditions: $94^{\circ} \mathrm{C}$ for $30 \mathrm{sec}, 54^{\circ} \mathrm{C}$ for $60 \mathrm{sec}$, and $72^{\circ} \mathrm{C}$ for $60 \mathrm{sec}$ for 30 cycles. To map $111 \mathrm{~m}$ (Zahedi et al. 1991), a 757-bp fragment from the $3^{\prime}$ region was amplified with the primers 5'-GGCCTGTAATAATCACCAAC- $3^{\prime}$ and 5'-CAGTTTAAGGCATCACACAA-3' under the conditions $94^{\circ} \mathrm{C}$ for $30 \mathrm{sec}, 55^{\circ} \mathrm{C}$ for $60 \mathrm{sec}$, and $72^{\circ} \mathrm{C}$ for $90 \mathrm{sec}$ for 30 cycles. The PCR products from the amplification of Pax8 and $111 \mathrm{~m}$ were resolved on HydroLink Mutation Detection Enhancement gel (AT Biochem), which allowed detection of a CAST:CBA heteroduplex product from heterozygous animals. For mapping of Bmi1, $1.1 \mathrm{~kb}$ of 3'untranslated sequence was amplified from mouse genomic DNA using primers $5^{\prime}$-GTGACTGTCGTCCAGTTTGC-3' and 5'-CCAGACGTTCACGTGAAGGC-3' and AmpliTaq Gold as described above, under the conditions $94^{\circ} \mathrm{C}$ for $30 \mathrm{sec}, 56^{\circ} \mathrm{C}$ for $90 \mathrm{sec}$, and $72^{\circ} \mathrm{C}$ for $120 \mathrm{sec}$ for 35 cycles. The amplified product was used to probe $B g l$ II-digested mouse DNA after gel electrophoresis and blot transfer. CAST DNA showed hybridizing fragments of 4.7 and $0.75 \mathrm{~kb}$, whereas CBA DNA had fragments of 4.7 and $1.5 \mathrm{~kb}$. To map nodal (Zhou et al. 1993) in the QTL analysis of chromosome 10, primers that amplified across an intron were designed $5^{\prime}$-CTCCACAATCATGTCCTTGTG-3' and 5'-GGCGAGTGTCCTAACCCTGTG-3' and used under the conditions $94^{\circ} \mathrm{C}$ for $30 \mathrm{sec}, 50^{\circ} \mathrm{C}$ for $90 \mathrm{sec}$, and $72^{\circ} \mathrm{C}$ for $120 \mathrm{sec}$ for 30 cycles. The amplified product from CBA DNA was $\sim 1200 \mathrm{bp}$, and from CAST $\sim 1030 \mathrm{bp}$, and were resolved on $4 \%$ NuSieve gels.

\section{DNA Pooling}

Phenotypically weighted DNA pools were generated by selecting backcross individuals at the extremes of tail-length distribution. Pool A was prepared from the $20 \% S d$ progeny $(n=30)$ with the shortest tails $(0-1.5 \mathrm{~cm})$, and pool B was prepared from the $20 \% S d$ mice $(n=30)$ with the longest tails $(2.5-4.8$ $\mathrm{cm})$. Five hundred nanograms of DNA per individual was used to generate the pools (Taylor et al. 1994).

\section{Analysis of Variance}

To assess the statistical significance of phenotypic variation in the backcross, we used the $F$ test on tail-length data obtained from $147 S d+$ and 144 wild-type backcross progeny of 24 days of age.

Assuming the null hypothesis that both distributions have the same variance; $F=s_{1}{ }^{2} / s_{2}{ }^{2}$, where $s_{1}{ }^{2}$ is the square of the larger standard deviation.

Given that the mean tail length in the $S d+$ population is $1.88 \mathrm{~cm}$ with a S.D. of 0.9755 , and that of the wild-type population is $6.975 \mathrm{~cm}$ with a S.D. of 0.6091 , an $F$ value of 2.56 with $n-1$ degrees of freedom yielded a highly significant $P$ value of $<0.00001$.

\section{QTL Analysis}

We mapped tail-length QTL to a number of chromosomes using a multiple regression method based on Haley and Knott (1992). In brief, the model considers a QTL (Q) positioned between two codominant flanking markers A and B, in a backcross population derived from two inbred lines, that is, $A_{1} A_{1} Q_{1} Q_{1} B_{1} B_{1}$ and $A_{2} A_{2} Q_{2} Q_{2} B_{2} B_{2}$. The two QTL genotypes possible in the backcross population have a mean effect of $m+d$ for $\mathrm{Q}_{1} \mathrm{Q}_{2}$ and $m-a$ for $\mathrm{Q}_{1} \mathrm{Q}_{1}$, where $m$ is the mid-parent (mean of homozygotes) and $a$ and $d$ are the additive and dominance deviation, respectively.

The recombination fraction $(r)$ between flanking markers is assumed known and is converted from genetic distance (in Morgans) by Haldane's mapping function (1919). For analysis, no interference is assumed; thus, $r=r_{\mathrm{A}}+r_{\mathrm{B}}-2 r_{\mathrm{A}} r_{\mathrm{B}}$, where $r_{\mathrm{A}}$ denotes recombination between $\mathrm{A}$ and $\mathrm{Q}$ and $r_{\mathrm{B}}$ between $\mathrm{Q}$ and $\mathrm{B}$.

The expected mean terms for putative QTL for backcross marker genotypes can be derived by summing over QTL genotypes and scaling for expected marker genotype frequencies (see Haley and Knott 1992). All calculations were carried out using Genstat 5.3 (Genstat 5 Committee 1993).

Numerical values of $a$ and $d$ for each marker genotype can be calculated for a putative QTL at a number of positions, for example, at 1-cM intervals between the flanking markers. Multiple regression allows numerical values of $m, a$, and $d$ to be fitted in a regression of the phenotypic measurement, providing an estimate for $a$ and $d$ at all putative positions. The likelihood ratio test was used to provide the test statistic likelihood ratio test $=n \log _{\mathrm{e}}\left(R S S_{\text {reduced }} / R S S_{\text {full }}\right)$, where $R S S_{\text {full }}$ is the residual sum of squares of the full model (with the QTL fitted) and $R S S_{\text {reduced }}$ is the residual sum of squares of the reduced model, where the QTL is omitted. Test statistic values were converted to lod equivalents by division with $2\left(\log _{e} 10\right)$. A $95 \%$ confidence interval for the QTL was determined by a one-lod drop (Lander and Botstein 1989).

Additional fitted effects in the model were a random effect of litter to account for common environmental variance of litter and a fixed effect of sex. The covariate of litter size and fixed effect of parity were not found to be significant in the model. QTL analysis was corrected for the effect of loci on different chromosomes by using marker loci as cofactors in the analysis (Zeng 1994). This allows each chromosome analysis to be considered independently. The percentage variance accounted for at each mapped QTL was estimated from the difference in the variance accounted for in the full model (with QTL) less that accounted for in the reduced model (omitting the QTL). 
ALFRED ET AL.

\section{ACKNOWLEDGMENTS}

We thank Vince Ranaldi and the staff of the Biomedical Research Facility for technical help with this project. We also thank Andrew Carothers for statistical advice, Wayne Frankel for helpful comments on the manuscript, and Nick Hastie for support. This work was initiated through a Medical Research Council studentship to J.B.A. and continues through a grant from the National Kidney Research Fund to I.J.J.

\section{REFERENCES}

Alkema, M.J., N.M. van der Lugt, R.C. Bobeldijk, A. Berns, and M. van Lohuizen. 1995. Transformation of axial skeleton due to overexpression of $b m i-1$ in transgenic mice. Nature 374: 724-727.

Beechey, C.V. and A.G. Searle. 1980. Chromosome 2: Distance of $S d$ from centromere. Mouse News Lett. 63: 17.

Conlon, F.L., K.M. Lyons, N. Takaesu, K.S. Barth, A. Kispert, B. Herrmann, and E.J. Robertson. 1994. A primary requirement for nodal in the formation and maintenance of the primitive streak in the mouse. Development 120: $1919-1928$.

Darvasi, A. and M. Soller. 1994. Selective DNA pooling for determination of linkage between a molecular marker and a quantitative trait locus. Genetics 138: 1365-1373.

Dietrich, S., F.R. Schubert, and P. Gruss. 1993. Altered Pax gene expression in murine notochord mutants: The notochord is required to initiate and maintain ventral identity in the somite. Mech. Dev. 44: 189-207.

Dietrich, W., H. Katz, S.E. Lincoln, H.-S. Shin, J. Friedman, N.C. Dracopoli, and E.S. Lander. 1992. A genetic map of the mouse suitable for typing intraspecific crosses. Genetics 131: $423-447$.

Dunn, L.C., S. Gluecksohn-Schoenheimer, and V. Bryson. 1940. A new mutation in the mouse affecting spinal column and urogenital system. J. Hered. 31: 343-348.

Genstat 5 Commitee. 1993. Genstat 5 release 3 Reference Manual. Oxford University Press, Oxford, UK.

Gluecksohn-Schoenheimer, S. 1943. The morphological manifestations of a dominant mutation in mice affecting tail and urogenital system. Genetics 28: 341-348.

Gluecksohn-Waelsch, S. and T.R. Rota. 1963. Development in organ tissue culture of kidney rudiments from mutant mouse embryos. Dev. Biol. 7: 432-444.

Gruneberg, H. 1953. Genetical studies on the skeleton of the mouse VI. Danforth's short-tail. J. Genet. 51: 317-326.

Haldane, J.B.S. 1919. The combination of linkage values and the calculation of distances between the loci of linked factors. J. Genet. 8: 299-309.
Haley, C.S. and S.A. Knott. 1992. A simple regression method for mapping quantitative trait loci in line crosses using flanking markers. Heredity 69: 315-324.

Johnson, K.R. and M.T. Davisson. 1996. Mouse chromosome 18. Mamm. Genome 6: S300-S308.

Koseki, H., J. Zachgo, Y. Mizutani, D. Simons-Chazottes, J. Guenet, R. Balling, and A. Gossler. 1993. Fine genetic mapping of the proximal part of mouse chromosome 2 excludes Pax8 as a candidate for Danforth's short-tail $(S d)$. Mamm. Genome 4: 324-327.

Kruglyak, L. and E.S. Lander. 1995. High-resolution mapping of complex traits. Am. J. Hum. Genet.

56: $1212-1223$.

Lander, E.S. and D. Botstein. 1989. Mapping Mendelian factors underlying quantitative traits using RFLP linkage maps. Genetics 121: 185-199.

Malas, S., A. Pilz, P.A. Lalley, L. Pate, D.A. Stephenson, and C.M. Abbott. 1996. The isolation and mapping of PCR markers specific to mouse chromosome 2. Mamm. Genome 7: $145-148$.

Plachov, D., K. Chowdhury, C. Walther, D. Simon, J. Guenet, and P. Gruss. 1990. Pax-8, a murine paired box gene expressed in the developing excretory system and thyroid gland. Development 110: 643-651.

Placzek, M., T. Yamada, M. Tessier Lavigne, T. Jessell, and J. Dodd. 1991. Control of dorsoventral pattern in vertebrate neural development: Induction and polarizing properties of the floor plate. Development (Suppl. 2): 105-122.

Siracusa, L., J.L. Morgan, J.K. Fisher, C.M. Abbott, and J. Peters. 1996. Mouse chromosome 2. Mamm. Genome 5: S22-S39.

Taylor, B.A. and S.J. Phillips. 1996. Detection of obesity QTLs on mouse chromosomes 1 and 7 by selective DNA pooling. Genomics 34: 389-398.

Taylor, B.A., A. Navin, and S.J. Phillips. 1994. PCR-amplification of simple sequence repeat variants from pooled DNA samples for rapidly mapping new mutations of the mouse. Genomics 21: 626-632.

van der Lugt, N.M., J. Domen, K. Linders, M. van Roon, E. Robanus-Maandag, H. te Riele, M. van der Valk, J.

Deschamps, M. Sofroniew, M. van Lohuizen, and A. Berns. 1994. Posterior transformation, neurological abnormalities, and severe hematopoietic defects in mice with a targeted deletion of the bmi-1 proto-oncogene. Genes \& Dev. 8: $757-769$.

Wallace, M.E. 1972. A single gene isolated from a modifier complex in the mouse. Genetica 43: 597-606.

46: 529 .

1976. A modifier mapped in the mouse. Genetica

Zahedi, K., M.F. Seldin, M. Rits, R.A.B. Ezekowitz, and A.S. 
Whitehead. 1991. Mouse IL-1 receptor antagonist protein. J. Immunol. 146: 4228-4223.

Zeng, Z.B. 1994. Precision mapping of quantitative trait loci. Genetics 136: 1457-1468.

Zhou, X., H. Sasaki, L. Lowe, B.L.M. Hogan, and M.R. Kuehn. 1993. Nodal is a novel TGF- $\beta$-like gene expressed in the mouse node during gastrulation. Nature 361: $543-545$.

Received September 9, 1996; accepted in revised form December $5,1996$. 


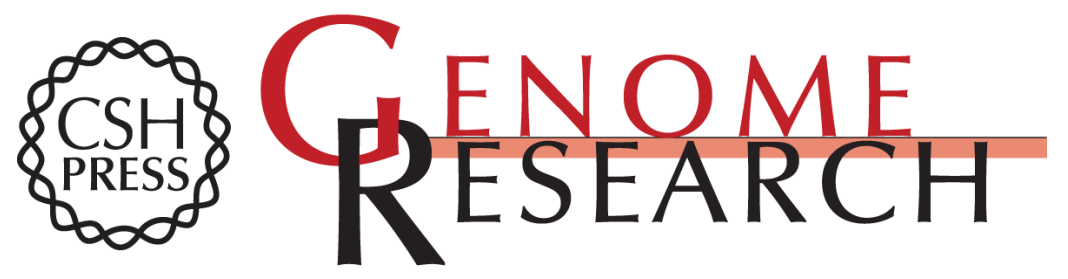

\section{Mapping in the region of Danforth's short tail and the localization of tail length modifiers.}

J B Alfred, K Rance, B A Taylor, et al.

Genome Res. 1997 7: 108-117

Access the most recent version at doi:10.1101/gr.7.2.108

References This article cites 27 articles, 9 of which can be accessed free at:

http://genome.cshlp.org/content/7/2/108.full.html\#ref-list-1

\section{License}

Email Alerting Receive free email alerts when new articles cite this article - sign up in the box at the Service top right corner of the article or click here.

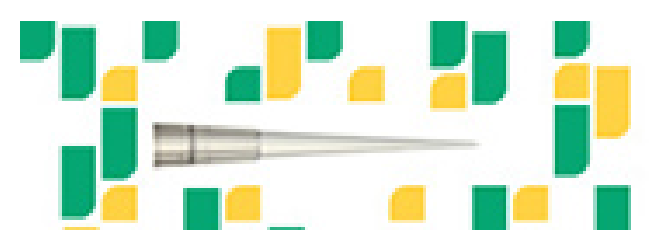

To subscribe to Genome Research go to: https://genome.cshlp.org/subscriptions 